\title{
RETROSPECTIVE STUDY OF 861 CASES OF ENDOSCOPIC ENDONASAL DCR: OUR EXPERIENCE
}

G. D. Mahajan ${ }^{1}$, Priya Shah², James Thomas ${ }^{3}$, Girija Ghate ${ }^{4}$, Mayur Ingale5, Paresh Chavan ${ }^{6}$, Anshuman Roy7, Kapil Duseja ${ }^{8}$

\section{HOW TO CITE THIS ARTICLE:}

G. D. Mahajan, Priya Shah, James Thomas, Girija Ghate, Mayur Ingale, Paresh Chavan, Anshuman Roy, Kapil Duseja, "Retrospective Study of 861 cases of Endoscopic Endonasal DCR: Our Experience". Journal of Evolution of Medical and Dental Sciences 2014; Vol. 3, Issue 09, March 03; Page: 2121-2125,

DOI: $10.14260 /$ jemds/2014/2113

ABSTRACT: AIM: To focus on difficulties in endoscopic endonasal DCR, to improve the final outcome of endoscopic endonasal DCR. To elaborate the steps this will avoid recurrence. MATERIAL AND METHODS: This article presents retrospective study of 861 cases that underwent Endoscopic endonasal DCR between Oct 2004 and Nov 2011. The patients operated were in the age group from 5 years to 94 years. The cases of lacrimal abscesses were tackled by endo DCR which gave a substantial advantage over the conventional external approach by avoiding a scar. The stenting of the canalicular system was restricted to the situations where the patency of the lacrimal canaliculi was absent and the sac syringing done on operation table showed no fluid coming from the new stoma due to the blocked canaliculi or fibrosis of lacrimal sac. The stent used was silicon bicanalicular lacrimal intubation set. CONCLUSIONS: Local anaesthesia preferred over general anaesthesia as it has less bleeding and less morbidity. Endoscopic DCR avoids scar of external approach. Coexistent sinonasal disease can be tackled at same sitting. Adequate marsupialization of sac mucosa is key for avoiding recurrence.

KEYWORDS: Dacryocystorhinostomy, Endoscopic DCR, Lacrimal abscess, Stenting.

INTRODUCTION: Epiphora due to blockage of the lacrimal drainage system is the most common symptom of chronic dacryocystitis. Dacryocystorhinostomy is done to provide alternative lacrimal pathway into the nose. The operative procedure can be done intranasally (Endoscopic Dacryorhinostomy - Endo DCR) or extra nasally (Conventional DCR).

Different techniques have been described for the Endo-DCR such as different flaps ${ }^{1}$, but in our study we have restricted to refashioning of the stoma with wide opening in the medial wall of the lacrimal sac.

Here the surgical procedure do not interphere the sphincter action of the lower punctum which in turn establishes the post-op function of the lacrimal system and these results in the satisfactory recovery of the patient with no facial scar.

Reduction in the morbidity, reduction in time taken for the surgical procedure and restoration of the physiological function are the prime features of the Endo nasal DCR.

MATERIAL \& METHODS: 861 cases between the age group of 5 years to 94 years with congenital or acquired NLD block visiting our hospital that underwent Endo nasal DCR were included in our study.

The general check up with fitness for anaesthesia was done for every patient. The patients with systemic diseases as Diabetes Mellitus, Hypertension, and heart disease were thoroughly investigated for fitness for surgery. The patients on anticoagulants were assessed and the anticoagulant medication was stopped before surgery. 
All patients were subjected to dacryocystography (DCG) and diagnostic nasal endoscopy (DNE). In pediatric patients, DCG, DNE and the surgery were done under general anaesthesia in single sitting only. In adults, DCG and DNE were done in outpatient department and the surgery was done under GA or LA depending on patient's condition.

The majority of the cases were done under local anesthesia with nasal packing with $4 \%$ lignocaine and adrenaline solution. 0 and 30 degree endoscopes were used. With local infiltration, the posterior based flap of mucoperiosteum was raised and was reflected posteriorly, the superior boundary of the flap at or just below the level of middle turbinate. The flap was elevated backwards and the part of the lacrimal bone and frontal process of maxilla were punched out. With the Kerrisons punch a bone window was created to visualize the lacrimal sac.

The lacrimal sac was exposed adequately and the medial wall of the sac was excised leaving the margins and lateral wall of the sac in situ. The common canaliculus opening could be visualized through the sac. Syringing was done through the pre anaesthetized lower punctum and the patency was assessed. The success was assessed by the free flow of the fluid in the sac syringing. In few cases there was no or restricted flow due blocked common canaliculus or the fibrosed lacrimal sac. In these select cases lacrimal stenting was done. The stent was kept in place for 6 weeks. Light nasal packing given to prevent postoperative hemorrhage.

POSTOPERATIVE CARE: The nasal pack was removed after 24 hours. Patients were discharged on oral antibiotics and decongestants, antibiotic steroid eye drops.

The patients were followed up regularly. Nasal endoscopy with sac syringing was done at each visit.

Postoperatively sac syringing was done twice a week, there after once a week for following 2 weeks and once in 15 days for next two months. Thereafter all patients were followed up monthly for one year.

RESULTS: Total 861 cases of congenital or acquired NLD block were operated for Endo DCR during the study period. In our study majority of cases were in the age group between 21 years to 70 years; out of 861 cases operated 735 were in this group (85\%).The male to female ratio was equal. Youngest patient was 5 years and oldest patient was 94 years. Main presenting symptom in almost all patients was epiphora. Out of 861, 12 patients presented with lacrimal abscess, 18 presented with lacrimal fistula. Complications such as primary hemorrhage, granulations formation, nasal synechiae formation, accidental opening of ethmoidal air cells were noted. But all these could be managed with minimal surgical interference.

Out of 861 cases Right side was operated in 457pts, Left sided DCR was done in 359 patients, and bilateral DCR was done in 45 patients.

Out of 861 patients, 12 (1.39\%) presented with lacrimal abscess and 18 patients (2.09\%) presented with lacrimal fistula and 135 patients (15.67\%) were operated under general anaesthesia. 115 (13.35\%) patients had coexisting sinonasal disease which was operated at same sitting. 2 patients had postoperative bleeding per nose because of rise in blood pressure which was stopped on control of their hypertension.

44 patients required revision surgery out of which 30 were operated outside our hospital and 14 patients developed restenosis. All 44 patients were subjected to silicon lacrimal cannulation. The patients who were cannulated did not have recurrence of symptoms. 
DISCUSSION: Since Toti described the initial dacryocystorhinostomy (DCR) operation in 1904 many technical modifications have evolved. ${ }^{2}$ Overall, three groups of procedures are currently practiced; external DCR, endoscopic DCR with contact laser, and surgical endoscopic DCR without laser.3,4

The Endo nasal DCR (Endo DCR) has a distinct advantage over the conventional External DCR, in that the facial scar is avoided. In the external DCR the medial canthal ligament needs to be excised which is involved in the pumping action, affecting the lacrimal drainage system. ${ }^{3}$

It is a simple, less time consuming, safe but skilful, highly satisfying surgery both for the patients as well as the surgeons. There is very big advantage of this surgery, it is close $100 \%$ successful procedure, even if there is recurrence of epiphora it is again correctable fully with no residual effects. ${ }^{5}$ The Endo nasal DCR is done by using the 0 degree endoscope. It gives an excellent illumination and better visualization of the operative field. This can be of an added advantage for wider exposure of the operative field.

The deviated nasal septum or any other local pathology can be tackled at the time of the Endo DCR surgery and due to the direct end on view. Marsupialization of the lacrimal sac can be done to the optimal size. The excision of the medial wall of the lacrimal sac to an optimal size gives a good marsupialization of the sac. The recurrence and the premature closure of the stoma can well be avoided by interposition of a specially designed Polyvinyl Acetol (PVAc) pack (Fig 2). In our experience the PVAc pack interpositioning has reduced the recurrence rate to negligible level.

The Endo nasal DCR has advantage over the Laser DCR as the puncti and the canaliculli are not exposed to the heat generated at the time of using laser. The heating may be responsible for the chances of the canalicular or punctum obstruction. ${ }^{6}$

The sac being directly visible and adequate removal of lacrimal bone and frontal process of maxilla avoids the injury to the lateral wall of the lacrimal sac and common canaliculus. This helps in maintaining the patency of the lacrimal system and high success rate may be attributable to this factor.

In our series, few difficulties were encountered, in the form of the very thick bone, which very hard to punch. Here we had to use the bone curette; sometimes the drill was used to drill the hard bone. In few cases there was DNS to the same side but we could manage the DNS and DCR procedures in the same sitting. Sometimes there was a noticeable bleeding which was controlled by local adrenaline packs. We had to abandon one case as we could not punch the bone to create the window to access the lacrimal sac. Occasionally the anterior ethmoidal cells were opened accidentally and further damage was avoided by restricting surgery limited to the lacrimal sac region. In rare instances of revision, the extra care was taken and the adequate size of the lacrimal mucosa was excised so as to have good patent stoma for lacrimal drainage.

The patients with lacrimal abscess were taken for surgery under antibiotic cover, and abscess was drained through the nasal cavity and incision was widened adequately for good drainage of the pus.

CONCLUSIONS: In our study majority of the cases were done under local anesthesia. This was helpful in lowering the morbidity and also helpful in avoiding the exposure of the patients to the anesthetic drugs. 
Endoscopic DCR is a better alternative to external DCR because also has a very minimal rate of recurrence and complications. Hospital stay is less so the loss of working hours is reduced to minimal level.

Coexisting sinonasal disease can also be managed simultaneously.

Due the anatomical familiarity of the ENT surgeon to that of the intranasal structures and the fluency of the handling the intranasal instruments, ENT surgeons stand for an added advantage.

The less number of stenting only suggests that adequate marsupialization is helpful to keep the stoma patent and avoid restenosis due to granulation formation.

The stenting of the lacrimal system should be restricted to the cases where easy flow was not established due to sac fibrosis and in revision cases.

The Endo DCR can be safely done in acute condition under the antibiotic cover. The Endo DCR when done for lacrimal abscess gives a safe and effective alternative method for draining abscess. Here the second surgery and the skin scar can be avoided and patient will have a fast recovery.

\section{REFERENCES:}

1. Sonkhya N, Mishra P. Endoscopic transnasal dacryocystorhinostomy with nasal mucosal and posterior lacrimal sac flap. J Laryngol Otol. 2009 Mar; 123(3):320-6. doi: 10.1017/S0022215108003897. Epub 2008 Oct 28.

2. Becker B.B. Dacryocystorhinostomy without flaps. Ophthalmic Surg 1988; 19:419-27.

3. Woog J J, Metson R, Puliafito CA. Holmium: YAG endonasal laser dacryocystorhinostomy. Am J Ophthalmol 1993; 116:1-10.

4. Jokinen K, Karja J. Endonasal dacryocystorhinostomy. Arch Otolaryngol 1974;100:41-4.

5. Sandeep Bhatnagar, Nirupama Singh, Vikram Lal. Endoscopic Endonasal Dacryocystorhinostomy: Best Surgical Management for DCR. Indian J Otolaryngol Head Neck Surg (October-December 2012) 64(4):366-369: DOI 10.1007/s12070-011-0345-0

6. Tsirbad A., Wormald P.J. Mechanical endo nasal Dacryocystorhinostomy with mucosal flaps $-\mathrm{Br}$ J Ophthalmol 2003; 87(I 0;43-47).

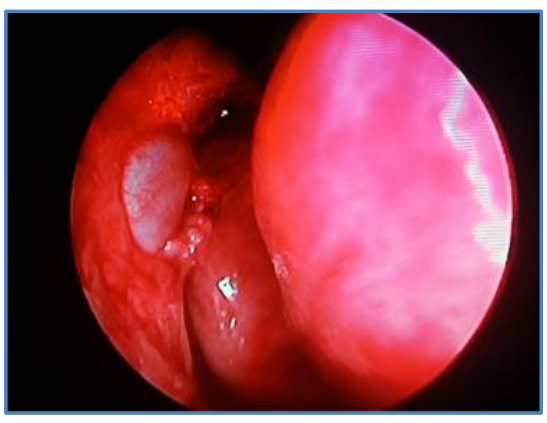

Figure. 1

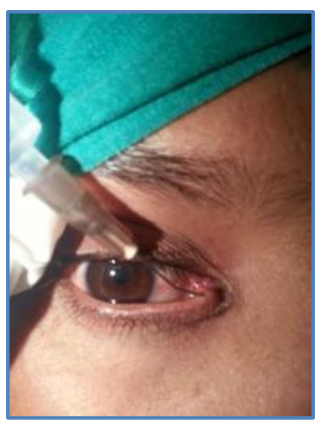

Figure. 2 


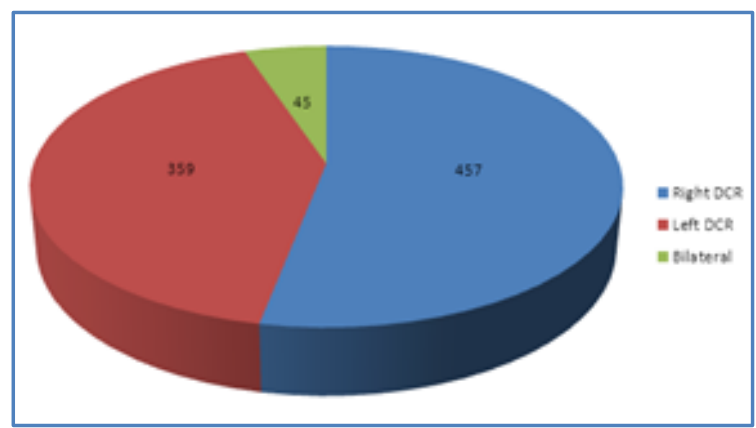

Table 1: Pie chart showing site of DCR

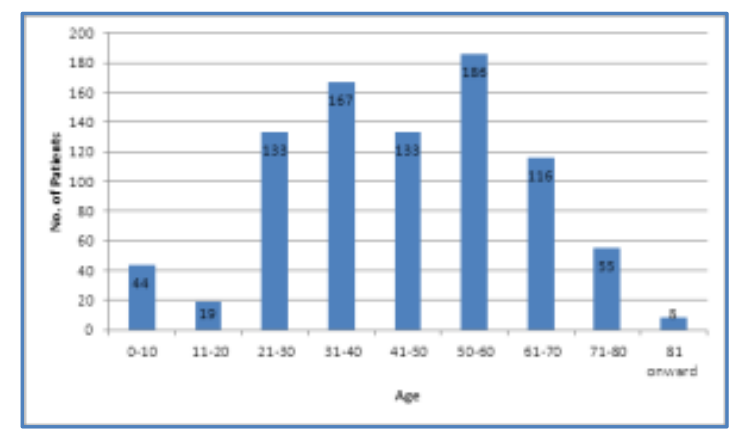

Table 2: Bar Diagram showing No. of patients in relation to age

\section{AUTHORS:}

1. G. D. Mahajan

2. Priya Shah

3. James Thomas

4. Girija Ghate

5. Mayur Ingale

6. Paresh Chavan

7. Anshuman Roy

8. Kapil Duseja

\section{PARTICULARS OF CONTRIBUTORS:}

1. Associate Professor, Department of E. N. T, PDM. Dr. D. Y. Patil Medical College, Hospital and Research Centre, Pimpri, Pune.

2. Assistant Professor, Department of E. N. T, PDM. Dr. D. Y. Patil Medical College, Hospital and Research Centre, Pimpri, Pune.

3. Professor and HOD, Department of E. N. T, PDM. Dr. D. Y. Patil Medical College, Hospital and Research Centre, Pimpri, Pune.

4. Resident, Department of E. N. T, PDM. Dr. D. Y. Patil Medical College, Hospital and Research Centre, Pimpri, Pune.
5. Resident, Department of E. N. T, PDM. Dr. D. Y. Patil Medical College, Hospital and Research Centre, Pimpri, Pune.

6. Resident, Department of E. N. T, PDM. Dr. D. Y. Patil Medical College Hospital and Research Centre, Pimpri, Pune.

7. Resident, Department of E. N. T, PDM. Dr. D. Y. Patil Medical College Hospital and Research Centre, Pimpri, Pune.

8. Resident, Department of E. N. T, PDM. Dr. D. Y. Patil Medical College Hospital and Research Centre, Pimpri, Pune.

\section{NAME ADDRESS EMAIL ID OF THE CORRESPONDING AUTHOR:}

Dr. Priya Shah,

A2/702, Kumar Princetown, Near California, NIBM Annexe,

Undri, Pune - 411060.

E-mail: drpriyashah@rediffmail.com

Date of Submission: 02/01/2014. Date of Peer Review: 03/01/2014.

Date of Acceptance: 03/02/2014.

Date of Publishing: 25/02/2014. 